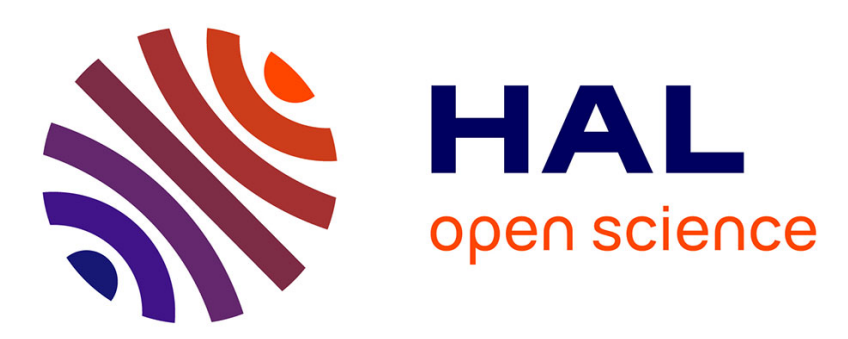

\title{
Building and Using Home Automation Systems: A Field Study
}

Alexandre Demeure, Sybille Caffiau, Elias Elias, Camille Roux

\section{To cite this version:}

Alexandre Demeure, Sybille Caffiau, Elias Elias, Camille Roux. Building and Using Home Automation Systems: A Field Study. ISEUD 2015, May 2015, Madrid, Spain. 10.1007/978-3-319-18425-8_9 . hal-01265223

\section{HAL Id: hal-01265223 \\ https://hal.inria.fr/hal-01265223}

Submitted on 2 Feb 2016

HAL is a multi-disciplinary open access archive for the deposit and dissemination of scientific research documents, whether they are published or not. The documents may come from teaching and research institutions in France or abroad, or from public or private research centers.
L'archive ouverte pluridisciplinaire HAL, est destinée au dépôt et à la diffusion de documents scientifiques de niveau recherche, publiés ou non, émanant des établissements d'enseignement et de recherche français ou étrangers, des laboratoires publics ou privés. 


\title{
Building and using home automation systems: a field study
}

\author{
A. Demeure ${ }^{1}$, S. Caffiau ${ }^{1}$, E. Elias ${ }^{2}$ and C. Roux ${ }^{2}$ \\ ${ }^{1}$ LIG, INRIA, Universités de Grenoble \\ alexandre.demeuredinria.fr, sybille.caffiaudimag.fr \\ ${ }^{2}$ Floralis, Universités de Grenoble \\ \{elena.elias, camille.roux\}@multicom-ergonomie.com
}

\begin{abstract}
These last years, several new home automation boxes appeared on the market, the new radio-based protocols facilitating their deployment with respect to previously wired solutions. Coupled with the wider availability of connected objects, these protocols have allowed new users to set up home automation systems by themselves. In this paper, we relate an in situ observational study of these builders in order to understand why and how the smart habitats were developed and used. We led 10 semi-structured interviews in households composed of at least 2 adults and equipped for at least 1 year, and 47 home automation builders answered an online questionnaire at the end of the study. Our study confirms, specifies and exhibits additional insights about usages and means of end-user development in the context of home automation.
\end{abstract}

Keywords: End User Development, Home Automation, field study.

\section{$1 \quad$ Introduction}

Ubiquitous computing has become a fact, even if in the different way than Weiser originally envisioned [1]. Widespread deployment of networks has supported interpersonal communication and enabled people to access information such as news and encyclopedias, as well as services such as GPS-enabled navigation systems and weather forecast. This range of services is now offered almost everywhere and at anytime via smartphones, tablets or even laptops and has become part of everyday life. Ubiquitous computing also takes place at home based on gateways such as ADSL modems and settop boxes, providing Wi-Fi local networking with high-speed connection to the Internet as well as rich multimedia services including TV, audio and video sharing. While this mostly represents how Ubiquitous Computing is currently taking place in households, a minority of them is also equipped with a home automation system.

There has been a recent trend in the past few years in the home automation domain: the emergence of radio based technologies (e.g. Z-wave ${ }^{1}$ or enOcean ${ }^{2}$ ) that enable households to be equipped with sensors and actuators in a way that is much more easy

\footnotetext{
${ }^{1} \mathrm{http}: / /$ www.z-wave.com/what_is_z-wave

${ }^{2} \mathrm{https}: / /$ www.enocean.com/
} 
to install and cheaper than previously available through wired solutions such as $\mathrm{KNX}^{3}$. Typically, it now becomes possible to get equipped with a home automation system (box plus sensors and actuators) from $€ 150$. A quite complete kit can be bought for about $€ 500$, which is comparable to the cost of a PC or a tablet. The improvements of their user interfaces and the support those boxes gives using several sensors/actuators technologies tend to lower some of the barriers identified in previous works [2,3] (cost of ownership, difficulty of preparing the infrastructure, inflexibility and poor manageability of the system) and transform the way people interact with the technology, which calls for new usability and usage studies.

While the literature on home automation systems users, uses and services is wide and varied, a quite complete and up to date review of these studies can be found in [4]. Our goal was to confirm, precise and get additional insights about why and how home automation box users do program their system.

In the rest of the paper, we first describe our protocol and households we recruited. We then discuss our findings and compare them to related works.

\section{Study}

This study took place in the east of France from spring to autumn 2014. We collected data from inhabitants who has been using a home automation box (such as the Zipabox, Zibase, Vera, eeDomus ${ }^{4}$ ) for at least one year in their home (at the time of the study) and can be considered as Do-it-yourselfers (as named in [2]).

By sampling participants as such, we aimed at getting an overview of current home automation real setups, the devices and services in use in such households, and their different usages. We also focused on the programs that inhabitants created to fulfill their needs via this system, in particular with regards to how they express such needs via the respective programming tools.

The study was conducted in 10 households, sampled and recruited from forums and researchers' acquaintances. It is composed of two parts: First, the technical referent (i.e. guru $(\mathrm{G})$ ) of the households answers an online questionnaire about the structure of the home (e.g. number of rooms), the identity of the inhabitants (age, technology habits, jobs...) and the home automation system characteristics (name of the box, when was it installed, kinds of sensors and actuators). The collected data defines participants' profiles, verifying their fitness in this study (...), as well as help prepare for the second part of the procedure.

A few days after the participant completes the online questionnaire, two members of the households (guru $(\mathrm{G})$ and companion $(\mathrm{C})$ ) were interviewed in situ for about 80 minutes. The interviews were video-recorded and photographs were taken after the participants gave consent. During the analysis phase, the videos were textually transcribed before being analyzed and interpreted. During the first 10 minutes, both guru and companion were asked to represent everything they considered as part of the automation

\footnotetext{
${ }^{3} \mathrm{http} / / / \mathrm{www} . \mathrm{knx} . \mathrm{org} /$

${ }^{4}$ http://zipabox.domadoo.com, http://www.zodianet.com, http://getvera.com, http://www.eedomus.com
} 
system in their home, using A4 paper and pencils. They were then asked to detail and explain the drawings in turn, which led to a description of their view of the installation. Although incomplete, this description highlighted the most significant home automation elements for household members. The semi-structured interview that followed concerned usages of their home automation system (with guru and companion) as well as technical installation and maintainability (with guru only). All participants were French-speaking and interviews were led in French. At the end of the interview, the participants received gift vouchers of $€ 80$.

\section{Participants}

Our study concerned households that can manage their home automation system by themselves (i.e. Do-it-yourselfers as named in [2]). In order to better profile these households, we first posted a link to an online questionnaire on home automation French-speaking forums ${ }^{5}$, asking for home automation systems that were in used as well as households characteristics (e.g. members, location). 47 persons (46 males and 1 female) freely answered the questionnaire. Analysis of the questionnaire allowed us to make a list of most commonly used home automation systems.

Table 1. Technical equipment of households.

\begin{tabular}{lcccc}
\hline Household & Name & Sensors & Actuators & Programs \\
\hline H1 & Zibase & $<5$ & $<5$ & $\sim 15$ \\
H2 & eeDomus & $\sim 20$ & $\sim 50$ & $>150$ \\
H3 & Crestron & $\sim 40$ & $\sim 40$ & $\sim 15$ \\
H4 & eeDomus & $\sim 40$ & $\sim 20$ & $\sim 30$ \\
H5 & Vera 3 & $\sim 15$ & $\sim 20$ & $\sim 30$ \\
H6 & Zibase & $\sim 15$ & $\sim 15$ & $\sim 60$ \\
H7 & HomeSeer 3 & $\sim 50$ & $\sim 50$ & $\sim 40$ \\
H8 & Zipabox & $\sim 15$ & $\sim 15$ & $\sim 15$ \\
H9 & Zibase & $\sim 15$ & $\sim 20$ & $\sim 60$ \\
H10 & eeDomus & $\sim 15$ & $\sim 15$ & $\sim 30$ \\
\hline
\end{tabular}

We then recruited 10 households that were composed of at least two adults, located in or around the south-east of France and equipped with one of the home automation system from the list. Due to location, only some of them were found via the first questionnaire, while others were recruited via forums and acquaintance. In addition to the adult members, 6 households were formed of a couple with 2 children, 1 household with 1 child and 1 household with 3 children. All households were house-owners and lived in a house (composed of 3 to 9 rooms) except the inhabitants of the 8th household,

${ }^{5}$ http://forum.eedomus.com, http://forum.micasaverde, http://fibaro.com, http://abavala.com/forums, www.touteladomotique.com/forum, Google+ communities (S.A.R.A.H, toute la domotique, Domotique Info). 
who live in a flat. Table 1 summarizes the technical equipment of the households (density levels of actuator, sensor and program are informal values from observation during house visits).

In each household, the guru happened to be male (data consistent with whom answered the online questionnaire). Due to their work domain or their training, 7/10 gurus were knowledgeable in computer science (cf. Table 2). No companion was found knowledgeable in computer science although 4 of them did have scientific training in biology or medical domains.

Table 2. Inhabitants.

\begin{tabular}{llccc}
\hline Guru & Age & Job & $\begin{array}{l}\text { Knowledge of } \\
\text { computer science }\end{array}$ & Companion's Job \\
\hline G1 & $41-55$ & Security agent & - & Factory worker \\
G2 & $26-40$ & Technical translator & X & Child-minder \\
G3 & $26-40$ & Electrician & X & Teacher \\
G4 & $26-40$ & Infrastructure security & $X$ & Interior designer \\
G5 & $41-55$ & Engineer & $\mathrm{X}$ & Health \\
G6 & $26-40$ & Computer Scientist & $\mathrm{X}$ & Realtor \\
G7 & $41-55$ & Manager & $\mathrm{X}$ & Engineer Biologist \\
G8 & $26-40$ & Technician & - & Nurse \\
G9 & $26-40$ & Railway technician & - & Administrative officer \\
G10 & $26-40$ & Computer Scientist & $\mathrm{X}$ & Manager \\
\hline
\end{tabular}

\section{$4 \quad$ Analysis}

In this section, we will first discuss roles and usages we found with respect to the literature. We will then present how households did choose their home automation system and present an installation overview of home automation in which every household we met can be projected. Last, we will present how these household members controlled and programmed the systems.

\subsection{Roles and usages}

Roles. Despite different types of participants (see Table 2), the relationship of household members to the home automation system was quite similar: First, only one member of the household was really interested in setting up and maintaining the home automation system, the male adult (which is consistent with the online survey we led: $46 / 47$ participants were males). The other adult member usually had a more distant relationship with the system, is not really interested what it can do and how, and do not 
want to spent time dealing with the technology. Actually, she considered the home automation as a hobby for her companion that they have to live with rather than a useful addition to the household. However, they are satisfied when the system works (they find it useful) and get frustrated when it does not.

At least one child lives in 8/10 interviewed households. While we did not interview them, none played an active role in future evolutions of the system, or its installation. The technology seemed to be adapted to them, so they became passive users and often a source of inspiration for gurus' scenarios programming. For example, G5 told us that he programmed the lights in the corridors to turn off automatically because his children often forget to do so.

With respect to [2], the roles identified in the households are gurus and consumers. No interviewed householder contained a resident who helped the gurus maintain the system (called assisters in [5]).

Table 3. Usages of home automation by interviewed households.

\begin{tabular}{|c|c|c|c|c|c|}
\hline & $\begin{array}{l}\text { Intrusion } \\
\text { detection }\end{array}$ & Monitoring & $\begin{array}{l}\text { Automatic } \\
\text { control }\end{array}$ & Heating & $\begin{array}{l}\text { Reminder } \\
\text { Notification }\end{array}$ \\
\hline H1 & - & Inhabitants (Camera) & - & $\begin{array}{l}\text { Shutters } \\
\text { Heaters }\end{array}$ & - \\
\hline $\mathrm{H} 2$ & - & $\begin{array}{l}\text { Inhabitants (Camera) } \\
\text { Energy consumption }\end{array}$ & Lights, portal & Heaters & $\begin{array}{l}\text { Outing the trash, } \\
\text { it's time to go at } \\
\text { school }\end{array}$ \\
\hline H3 & Camera & - & Lights & Shutters & - \\
\hline $\mathrm{H} 4$ & Alarm & Inundation, smoke & $\begin{array}{l}\text { Swimming } \\
\text { pool (filtra- } \\
\text { tion) }\end{array}$ & Heaters & Sensors' state \\
\hline H5 & $\begin{array}{l}\text { Alarm, pres- } \\
\text { ence simula- } \\
\text { tion }\end{array}$ & - & $\begin{array}{l}\text { Climate, } \\
\text { shutters, } \\
\text { lights }\end{array}$ & Shutters & - \\
\hline H6 & Camera & - & Lights & $\begin{array}{l}\text { Heaters } \\
\text { Shutters }\end{array}$ & $\begin{array}{l}\text { Presence detection } \\
\text { (children, guests) }\end{array}$ \\
\hline $\mathrm{H} 7$ & Camera & $\begin{array}{l}\text { Energy consumption, } \\
\text { Temperature, humidity }\end{array}$ & $\begin{array}{c}\text { Lights, } \\
\text { swimming } \\
\text { pool (filtra- } \\
\text { tion) }\end{array}$ & $\begin{array}{l}\text { Heaters } \\
\text { Shutters }\end{array}$ & - \\
\hline H8 & Alarm & Energy consumption & Shutters & Heaters & - \\
\hline H9 & $\begin{array}{l}\text { Camera, } \\
\text { presence de- } \\
\text { tection }\end{array}$ & - & Lights & $\begin{array}{l}\text { Heaters } \\
\text { Shutters }\end{array}$ & - \\
\hline $\begin{array}{l}\mathrm{H} \\
10\end{array}$ & $\begin{array}{l}\text { Camera, } \\
\text { alarm }\end{array}$ & $\begin{array}{c}\text { Inhabitants (Camera) } \\
\text { Temperature } \\
\text { Inundation }\end{array}$ & Lights, kettle & - & $\begin{array}{c}\text { End of the laundry } \\
\text { cycle }\end{array}$ \\
\hline
\end{tabular}


Usages. The reasons why interviewed households acquire home automation boxes are consistent with literature [3, 4, 6]: Primary motivations are related to energy saving, automatic control and security (intrusion detection). Once this first objective is satisfied by a first installation, evolutions are performed in order to improve the first objective or to take into account new ones (e.g. Activity monitoring, notification). According to the motivation "Experiencing Benefits Increases Interest in Upgrades" [3] several of the evolutions are opportunistic: Gurus create new usages because they found themselves in a situation in which they found home automation systems useful. For example, G2 and G10 used their cameras (installed for intrusion detection) to watch their children play in the living room while lying down in their bed on Sundays.

Table 3 summarizes the usages of home automation by the interviewed households. H5 and H8 wanted to achieve a state of peace of mind as identified by [2, 3, 7]. H2, H3, $\mathrm{H} 6$ and $\mathrm{H} 7$ were more focused on comfort by delegating some domestic tasks to the system. For G2, these tasks were repetitive, time-consuming and/or unrealizable when household members are not present. To achieve its goal, the home automation system must be applied in a non-intrusive manner. As a consequence, G2 tried to make the system as autonomous as possible so that it acts without needs for others inhabitants to explicitly interact with it. "It is also what makes my wife feel the home automation less imposing or perceivable because once it is in place, the rules are almost... Finally I would say we no longer modify the implementation, as the home lives its life by itself".

\subsection{Choice of the home automation system}

Choosing your home automation system is a task only the guru performed. All the interviewed gurus looked for information about several boxes before buying theirs. They found information on dedicated websites or forums where existing users share their experiences. Four main criteria are cited: economic (price of the set-up box and available sensors and actuators), origin (national product or not) (H9), ease of installation and ease of maintenance. Future users often check compatibility with all connected objects already present at their homes or that they plan to add. For instance, G1 wanted the system to be able to pilot his shutters. Most of the gurus also expressed their concern about having a box able to deal with as much as protocols as possible.

Three households changed their home automation box to migrate toward a more up to date hardware or more powerful and easy to use systems (H2, H4 and H7). However, most of the evolutions concerned adding or removing devices and services. From interviews, we identified three reasons to make the home automation system evolve. First, like all technical installation, home automation system components deteriorate with time so sensors and actuators have to be replaced. Second, in order to fulfill new needs, devices and services may be added. Third, as pointed out by [3], an important motivation for guru is about managing the home automation system, experimenting new devices or services as a hobby.

Evolutions of the home automation system are planned, organized and technically installed by the guru. C2, C4 and C6 intervened in this process to modify an initial installation. For example, $\mathrm{C} 2$ asked her husband to switch off the vocal reminder when children are not awake. Moreover, when they plan to include new equipment, or to 
program a new functionality, gurus usually try to take into account their wife's needs. For example, G7 said he considers the Wife Acceptance Factor (WAF) of any equipment before buying it. That is to say that his wife has to accept the inclusion of the device, considering esthetical aspects but also that the targeted usage will be at least tolerated by her.

\subsection{Installation overview}

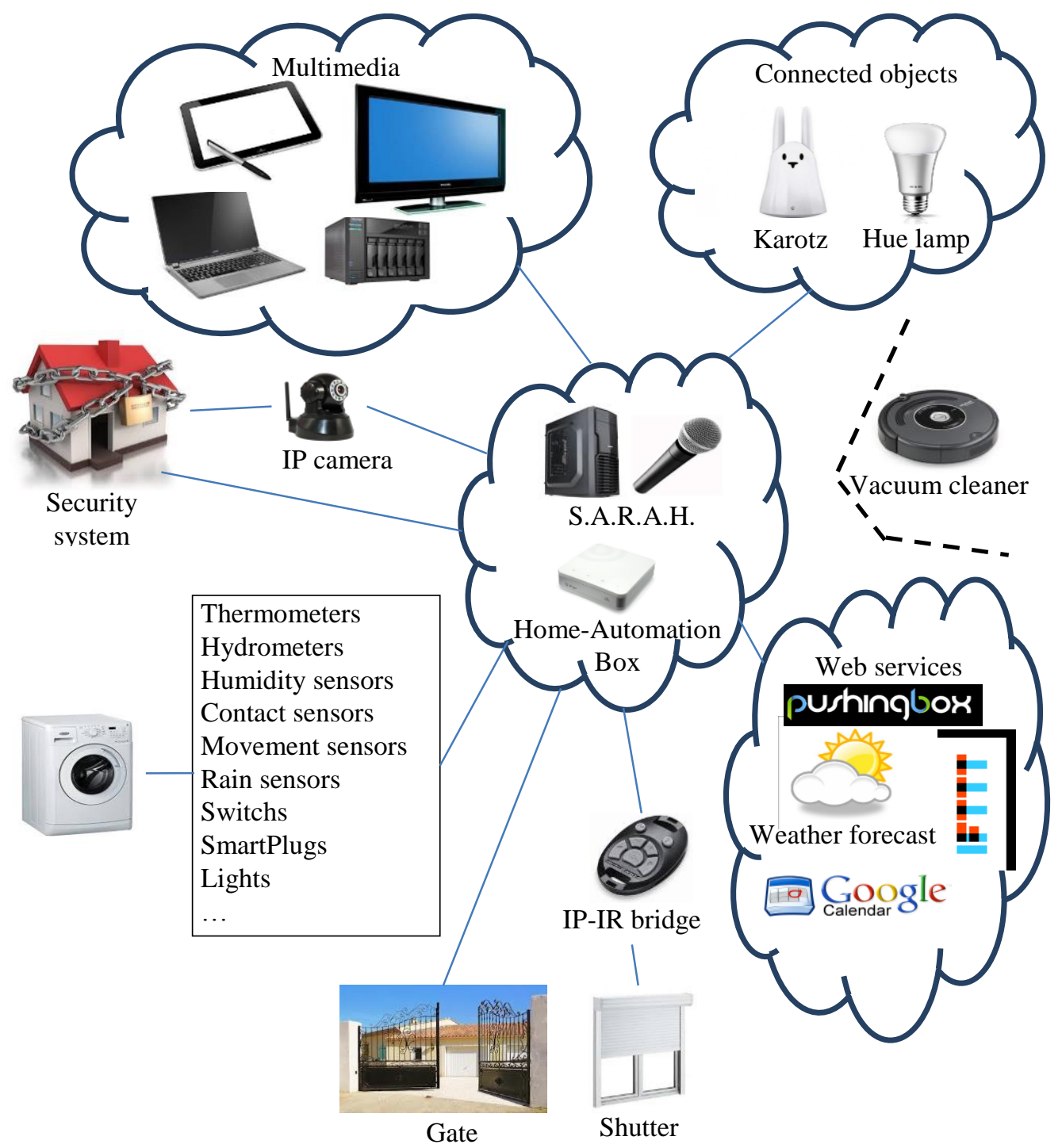

Fig. 1. Devices, services and their connections. 
With respect to [2], we found that the media controller was not considered as being part of the home automation system although every household was equipped with advanced media controller. The reason may be that multimedia systems are now widespread: almost every new TV is UPnP compliant and setting up a NASS system is quite common. The other reason is that multimedia systems can work alone, there is no technical need to weave it with into the home automation system, it appears as a separate system, although communications with home automations are possible and sometimes done.

Fig. 1 illustrates the devices we observed in participant's household as well as their connections. No one had all these devices but every participant household can be projected in this schema. What participants tended to consider as home automation is the home automation box coupled with sensors and actuators, automatic gates, shutters. H2, H7, H9 and H10 installed S.A.R.A.H. (or planned to do so soon) in addition to their box. This software enables participant to use voice recognition in order to control their actuators as well as multimedia systems or connected objects. S.A.R.A.H. was installed on PC distinct to the home automation box but accessed sensors and actuators through the box.

Every participant had a multimedia system enabling video streaming between devices (often based on UPnP). Although every box was able to handle multimedia services, height households did not integrated it with the rest of the home automation. They indeed did not need such an integration, as their usage of multimedia and home automation was clearly separate. They were using their multimedia system via dedicated software such as $\mathrm{XBMC}^{6}$. However exceptions were found first in household equipped with S.A.R.A.H., who used it to control multimedia rendering. H7 programmed some lights to be turned on when a TV was turned on. $\mathrm{H} 2$ used it to help the family routine along: music was played in the bathroom at 7:00 then in the kitchen at $7: 15$, following the family routine.

Situation was similar for automatic vacuum cleaner: Four participants had such a device but only $\mathrm{H} 2$ tried to integrate it with the home automation system, without success because of API problems. His goal had been to trigger the cleaning process when family members were out.

Seven participants had a subsystem dedicated to security. G3 clearly separated it from his home automation system for security reason. He managed the security system via its dedicated software. For the other participants (H2, H4, H6, H7, H9, H10), security was achieved using sensors, shutters and IP cameras. For the later ones, both home automation system and dedicated software were used.

We found some connected objects in participants' household. The most popular was Karotz, present in seven households. It was, or had been, mainly used to notify inhabitants (e.g. "it's time to go to school" or "today wastes are taken out") or as an aesthetic camera+microphone+loud speaker device (e.g. one participant used it to check whether his child was doing homework from his workplace, eventually launching a "do your homework" notification to the child). However, due to the recent decision of the Violet

${ }^{6} \mathrm{http}: / /$ kodi.tv/ 
Company to stop the support, some participants simply stop using it. Others turned toward Open-Karotz, an alternative open-source solution.

Last, six participants ( $\mathrm{H} 1, \mathrm{H} 2, \mathrm{H} 4, \mathrm{H} 5, \mathrm{H} 7$ and $\mathrm{H} 8)$ has been interoperating their home automation system with google calendar. This was due to its availability everywhere online, their previous familiarity with the application, often considered better than other calendars provided by their home automation system in every aspect. Other web services were also used such as IFTTT ${ }^{7}$, weather forecast and pushing box to send notifications.

\subsection{Controlling the system}

We observed that inhabitants were interacting with the system throughout 5 interfaces, namely: PC, smartphone, tablet, Karotz, S.A.R.A.H. (vocal command) and dedicated remote controllers for shutters or multimedia. According to our online questionnaire, $36 / 47$ gurus daily interact with the system via a PC or a smartphone. Main usages were for monitoring energy consumption (25/47 use PC and 23/47 use SmartPhone), monitoring system state (39/47 use PC and 28/47 use SmartPhone) and controlling devices (31/47 use PC and 42/47 use SmartPhone). Programming scenarios was achieved almost exclusively using the household's personal computer or laptop (40/47).

Households equipped with S.A.R.A.H. use it to control multimedia (H9), lights and shutters $(\mathrm{H} 2, \mathrm{H} 7)$. H2 also used it as a timely reminder for children to go to school. Overall inhabitants that used S.A.R.A.H. were satisfied, but they expressed concerns related to false positive detections. $\mathrm{H} 9$ and $\mathrm{H} 7$ had to uninstall the system when it was unable to recognize vocal command of the female inhabitant.

Karotz was used the most to send vocal notifications. For instance, G4 says us: "every morning at 7h45am, if the temperature is less than $5^{\circ} \mathrm{C}$ then it [Karotz] says: "be careful it's cold outside",". It has to be noticed that the producer of Karotz recently stopped its support so households had to turn toward open source solution or stopped using it completely.

Last, consistently with [3], all households told us that they considered very important to keep usual ways of interacting with the home such as light switch, remote controllers and switches for shutters and multimedia. Two reasons were cited: First, inhabitants anticipated difficulties of guests confronted to non-standard interaction technologies. Second, they felt more comfortable with the idea of having "traditional" backups as the home automation system turned to be sometime unreliable.

\subsection{Scenarios / Programs}

We asked participants to present their scenarios (sometime called rules) in order to get a better understanding on how they were programmed. The first finding was the diversity of strategies for naming scenarios. G8 both named scenarios with respect to the device involved (e.g. Plug2), the actions to be triggered (e.g. rain alert), the state of a device to be reached (e.g. full open) or a goal to be achieved (e.g. comfort). Seven

${ }^{7}$ https://ifttt.com/ 
gurus used the system's ability to group rules for factorizing conditions (e.g. sunset) or for expressing semantic proximity (e.g. all rules related to lights timeout). G10 named groups with respect to the environmental property of the targeted change (e.g. Light, Heating). For the Heating group, rules were named with respect to the schema DAYTYPE - ACTIONCONDITION, where DAYTYPE was either a week day, weekend or holiday while other groups where only named by combining related conditions and actions. DAYTYPE was actually used as a way to contextualize rules such as "turn on heating in the morning". Last, H6 used professional naming convention for his scenarios, prefixing names by '\#' when scenarios were dedicated to configure devices, '@, when it concerned the system's reaction to events and ' ', when scenarios had to be hidden from user interface (equivalent to the private attribute in object programming). ECA structure. Contents of the scenarios were either: 1) configuration instructions for a device (e.g. to set up measurement frequency for sensors), 2) scenes (i.e. a configuration of actuators) that could have been defined either by programming or by example or 3) Event Condition Action (ECA) rules. For this later, subtle differences differentiate boxes of the participants:

- ZiBase offers a simple ECA structure, it is possible to specify one event (called stimuli), to express conditions with conjunctions and disjunctions (called criterions) and a list of actions that may contain call to other rules.

- eeDomus offers a slightly different structure. Rules may contain 4 parts: 1) temporal conditions (called schedules), 2) events and conditions other than temporal (called Criterions) that can be combined with conjunctions and disjunctions 3) actions that may contain calls to other rules and 4) notifications such as mailing, texting, etc...

- HomeSeer enables users to regroup rules and to factorize conditions and actions via these groups. Rules have a WHEN THEN structure, the WHEN part containing conditions and events combined through disjunctions of conjunctions. It is also possible to trigger events in the THEN part. For some specific case, G7 wanted to use conditions intertwined. As it was not possible via Homeseer, he used VisualBasic to program the functionality instead.

- Zipato rules are based on Skratch [8] and enable users to specify quite complex instructions flows, although G8 regretted the absence of control loop such as "do this every N seconds". Rules start with a "when" statement that specify the event which will trigger the rule. IF THEN ELSE and REPEAT structures can be used inside the rule to control instructions execution. While powerful, this can also turn complex. G8 had to use advanced instructions such as "join" (stop other executions of the same rule) and "stop" (stop this execution of the rule) in order to make one of his program work, even though he clearly stated that he did not really understand the meaning of these instructions.

- Programming with Vera is more scene-centered. Users define scenes (devices configuration) and can associate triggers and schedules to them. Conditions cannot be directly expressed inside scenes. Vera enables users to use $\mathrm{Lua}^{8}$ language to express more complex scenarios.

\footnotetext{
${ }^{8}$ http://www.lua.org/
} 
- Last, Creston offers an application so complicated to program (or even read) rules that we were not able to evaluate it subsequently. The rules used by $\mathrm{H} 3$ were complicated to set up even though he was helped by a friend whose job was to install such systems. We can only say that it seems to be based on ECA rules.

Importance of time. We observed the importance of time in scenarios. All the participants dealt with some time-based rules, for instance to pilot heating system depending on week days, week-ends or holidays. Many participants were not plainly satisfied with the temporal representation offered by their home automation system to trigger rules. As a consequence, they managed to interface with google calendar, either by using dedicated plugins (everyone but H8) or by deploying a google script polling the box with current calendar events (H8). Reasons expressed by participants for doing so lied in their continuous personal and/or professional use of google calendar and wide-spread availability unlike the one proposed by their home automation system.

G2 programmed a quite specific morning scenario, playing music first in the bathroom, then in the kitchen $15 \mathrm{mns}$ later. Based on the clock, vocal notifications triggered for kids to go to school and, depending on the day, for throwing the trash. G6 used timeouts to open or close his shutters sequentially as doing it simultaneously led to blowing fuses. G7 used timeouts to turn lights off 1 minutes after any movement was detected by the related sensor. He also considered duration of state to prevent his shutters opening and closing several times at sunset (which is an hour managed by the box, as sunrise): "if the luminosity has been less than 600lux for 10 minutes, then close the shutters".

Usage of dedicated modules. In most boxes, it was possible to use modules (i.e. functions) to alleviate scenarios programming. For instance, G1 used in his scenarios a modules provided by Zibase to pilot his thermostat. He first tried to implement the desired behavior by himself but encountered problems due to hysteresis consideration, and therefore adopted the module as soon as it was made available, needing only to configure it for his needs. eeDomus also propose such a template mechanism called "programmation" (ie: programming). A programmation is a pre-specified scenario that users have to instantiate and configure for their needs.

For all home automation systems, modules appear as black boxes and are available through constructor websites or directly on the market. We observed that most of the modules were proposed by other users who were expert enough to program them using more advanced languages, such as Lua or Visual Basic. This stresses the importance of the community of users for novice households. Indeed, current or future users help each other to choose home automation box, and deal with installation or programming problems. All of our participants used forums, either as mere readers or as active participants. G5 uses to frequent forum to help other users or exchange with module developers. For instance, he talked profusely with the creator of a VMC manager module, helping to debug it, providing logs and hints. He really enjoyed this experience: "there is often a lot of follow-up, people are having fun helping others, which is nice". 
Importance of interoperability. Except for Creston, all boxes offered some kind of interoperability with other services or devices, mainly through dedicated modules or the possibility to send and receive HTTP requests. G8 was probably the participant that used HTTP the most, although he was not familiar with networks beforehand. His first motivation for using HTTP was to avoid buying dedicated modules for integrating Karotz into his scenarios. He also used HTTP request inside scenarios to send SMS throughout the household set-top box, send notifications via the pushing box web-service, pilot his IP cameras or get informed about electricity rate changes (night/day). Symmetrically, with the help of a friend, he set up google scripts to get interface his calendar with the box, sending HTTP requests to his box containing current agenda events. Other participants used HTTP request to interoperate with external services, for instance G10 used the weather forecast web-service of his town with X-PATH queries to retrieve relevant data.

Another kind of interoperability was about managing shutters and gates as some of them did not offer open APIs. A workaround was to use IP to IR (infrared) devices that were taught association between IR signals and HTTP requests. From the home automation box perspective, this was integrated through the definition of virtual devices.

Variables and virtual devices. Seven gurus defined variables for their programs. These variables aimed at representing states (e.g. is the home occupied?), specific values (e.g. how many degrees represent cold or warm for the heating system), virtual devices (e.g. defining a tailored alarm aside from the one provided by the box by waving together Karotz, as well as a dedicated alarm system and contact sensors). Except in the case of $\mathrm{ZiBase}$ and Creston boxes, it was possible for gurus to specify the name of variables which helped users to make sense of them and to use them inside scenarios.

Comments. We found that although rules were most of the time expressed using pseudo natural language, the meaning was not clear enough for participant to explain them to us in return. Some participants explicitly stated that there was a lack of commentary support. The situation was even worse for ZiBase users that have to deal with variables named V1 to V32. G6 and G9 had to maintain a Microsoft Excel ${ }^{\mathrm{TM}}$ stylesheet aside their box in order to remember the meaning of each variable.

Comments were also lacking when participants were debugging/tuning their scenarios. For instance, G10 spent time to find the correct detection threshold allowing his motion sensor to ignore the cat that occasionally walks around the door and didn't need the lights. He had to try multiple values for the cat to be ignored while the children weren't for instance. Threshold values were noted aside from the system as there was no commentary support available.

\subsection{Testing}

The most common way the gurus tested programs was by trial and error, running the program and observing the resulting behavior(s) within the house. However some prob- 
lems occurred a long time after the program was set up. For instance, two of the households ( $\mathrm{H} 2$ and $\mathrm{H} 7)$ did mention that they had to reprogram their shutter for them not to close when night is detected while the corresponding French-window is open. Indeed, they experienced summer evenings where inhabitants stayed late in the garden and found the shutters suddenly closed (originally to keep home temperature ideal). When undesired behavior was reported (by guru or another member of the household), the guru endeavored to debug and fix it. This may mean looking in the system's traces whether messages from sensors were received (which may happen when a sensor battery is low). Traces are also used preventively when programming to check whether rules were triggered, actions performed or events received, even by gurus that do not have computer science training (e.g. G1 used traces to debug his Karotz).

Testing the completion of actions was sometimes rendered impossible. This was the case for G9 who wanted to check whether shutters were opening or closing during the day: During winter, both G9 and his wife go to work before sunrise and come back after twilight so it was not possible for them to check by themselves whether everything happened as programmed. As a consequence, G9 programmed notifications to be sent on his phone whenever shutters were opened or closed, which reassured him even after debugging was done.

In the same vein, testing scenarios sometimes implied to shorter evaluation delays. For instance G9 shorten its heating scenario evaluation frequency from every 10 minutes to every 10 seconds in order to test Hysteresis thresholds. G10 also had to modify the energy consumption measurement frequency of a smart plug alimenting his washing machine in order to be able to detect when it really stopped.

Another strategy was to set up a virtual device to simulate real one and see what happen when setting up specific values. For instance, G5 simulated temperature changes to validate his heating scenarios.

Some boxes provided a test button (eeDomus, ZiBase) associated with scenarios. It actually triggered the ACTION part of the rule, allowing the guru to validate the action. However, it turns to be insufficient as pointed out by G6 for whom it was really difficult to program the EVENT-CONDITION part of scenarios. Indeed, finding out the right sensor values or the right conditions turned out to be the real challenge. G10, for instance, spent time to tune his heating scenarios to take into account holidays, sunset and twilight, sensed lights, presence detection and so on.

A more useful functionality, proposed by a couple of boxes (eeDomus, HomeSeer) was the possibility to navigate between rules and their associated devices or services. This was used by G10 to preventively check the impact of a modification (e.g. removing a sensor) on the system. G8 also used it to turn off scenarios that impact devices in use in scenario he wants to add. For instance, he disenabled scenarios controlling lights when he wanted to test a newly bought light.

\section{Discussion}

Unlike what was observed in [2] and [3], only two of the households we interviewed did equip their homes with automation systems after major renovations (H7) or building 
a new home (H9). The gurus of these two households were not able to find skilled professional able to integrate home automation system with the heater (H9) or the electric and data networks (H7). They had to deal with the installation by themselves, which turned to be quite difficult for the heating system of H9. However, H7, H9 and other households took advantage of the fact that their automation system used radio protocol to deploy the home automation system with minor changes to the home: "only" aesthetical consideration had to be taken into account so that other household members did not reject the system outright. This tends to confirm that the evolution of home automation technologies (radio protocols) and the lower costs enable more people to equip with automation systems.

We found that the fundamental motivation of gurus to equip their household was related to their hobbies. Most of them follow the news about home automation, frequent dedicated forums, try to be up to date. The decision to get the technology was often made because of the availability of the new generation of home automation system. Lowering energy consumption, improving security (intrusion detection) or comfort are also real objectives but serve more as a justification for others. This means that most of them are happy to spend time installing and tuning their home automation system. G8 even regretted that it was sometimes too easy, talking about its tailored alarm system: "I was almost frustrated because I did it within 2 minutes and in the end I did not enjoy fiddling with the system, it was almost too simple”.

Seven of the gurus we interviewed had some background in computer science, which may explain why they did not encountered extensive problems in programming. However even G1, G8 and G9 who do not have such a background, were able to program their scenarios and make them work. All programming system we considered are based on the Event-Condition-Action (ECA) paradigm, as already pointed out by [9] and [10], peoples are able to use it to express what they want. However, we found that home automation systems provide quite different ways to express ECA rules, it would be interesting to further investigate on forums the advantages and disadvantages of each as perceived by users. One limit that we identified was about grouping rules (and variables) related to a same objective into a consistent object. The roundabout way to deal with that is naming related rules with a same prefix but it does not provide a higher level of abstraction (and understanding) for gurus. We think that some effort should be put in providing ways for gurus to build higher-level abstraction from rules, variables and devices. Virtual devices may be a way to tackle that problem, activity may be another one [11].

It has to be noticed that programming is not limited to specifying ECA rules, some gurus made use of more generalist languages such as Visual Basic, Lua or even google scripts (JavaScript). Of course, only gurus with quite knowledgeable in programming made use of them. What really surprised us was how widespread the usage of HTTP APIs, provided by online services (e.g. weather forecast) or Karotz, was. G8 is the most significant case for that. Although he had no training in computer science, once he understood the principle of HTTP request, he was able to use them numerous times in his programs to communicate with Karotz, online services, his IP to IR bridge and even google script. Every system we considered did offer a way to send HTTP request, some also offer ways to specify HTTP request to be received (by specifying a virtual device 
as for G8). It seems to us that it is a quite simple and powerful way to make things communicate inside the home, even for non-professional programmers.

Current home automation systems lack of support for conception. This manifested through the inability for gurus to enter comments related to the threshold values they use, the meaning of their variables or the raison d'être of some rules. More generally speaking, we think that home automation systems should provide support for the conception process: expressing needs, considering options, discussion, adding tests, bug tracking etc. In some sense, this is close to Mennicken's claims [3] for the necessity of taking into account the different stages for creating a smart home. These stages would have to provide support for higher level goal or even for expressing household values $[11,12]$ and could lead a first step for integrating other household members who currently prefer staying aside. Indeed, gurus' companions seemed to be mere passive users of the system and this is somehow surprising when considering that they have been literally living inside the system for at least one year. This may be due to a lack of interest in that kind of technology but also on the feeling of being unable to take control of it. For instance, C2 expressed her desire to disable smartphones and TV during the dinner time, therefore using the system to enforce a family rule but she just thought that it was impossible. Other companions manifested interest when talking about high-level goal (e.g. managing stocks, keeping the home quiet when the baby was sleeping).

Last, we have to stress the importance of home automation online communities that exist around dedicated websites and forums. While gurus are currently quiet isolated geographically (only G9 physically knew someone with who to talk about home automation), these online communities are the main source of information as to give and share advices about systems, devices, installation problem, etc. It represents also a source of inspiration of possible usages and a place to discuss feedbacks. As pointed out by [4] and observed on services like IFTTT, rules created by the community can be proposed to gurus in order to inspire them. What we suggest is that not only rules should be made available in such a way but also discussions, goals, problems and solutions, etc. In other word, we suggest that it would be interesting to tackle end user development for the smart home from a social perspective and build tools accordingly.

\section{Conclusion}

We presented a study about current households equipped with a home automation system installed and managed by inhabitants themselves. We interviewed 10 households composed of at least 2 adult members that have lived with their system for at least one year. Our goal was to confirm, precise and get additional insights about why and how home automation box users program their system.

We observed that roles of gurus and consumers are defined before the introduction of the home automation system and they do not seem to change over time. We established the topography of devices and services and noticed that there were similarities across households. We also noticed that vocal interaction was getting popular via S.A.R.A.H. 
We compared the programming languages proposed by the different systems, all are based upon ECA structures but presenting subtle differences. The interoperability between sensors, actuators, and connected objects does not seem to appear difficult to use by the gurus when taking into account their choice of box. Thanks to forums and online communities, they learn how program and capitalize on the features of their box.

Based on these observations and the conclusions of related works, we presented several research avenues for home automation system in the discussion.

Acknowledgements. This work has been supported by the European Catrene project AppsGate.

\section{$7 \quad$ References}

1. Rogers, Y.: Moving on from weiser's vision of calm computing: engaging ubicomp experiences. UbiComp'06. pp. 404-421 (2006).

2. Bernheim Brush, A.J., Lee, B., Mahajan, R.: Home Automation in the Wild: Challenges and Opportunities. CHI 2011. , Vancouver, Canada (2011).

3. Mennicken, S., Huang, E.M.: Hacking the Natural Habitat: an in-the-wild study of smart homes, their development, and the people who live in them. Pervasive'12. pp. 143-160 (2012).

4. Mennicken, S., Vermeulen, J., Huang, E.M.: From Today's Augmented Houses to Tomorrow's Smart Homes: New Directions for Home Automation Research. UBICOMP'14. pp. 105-115., Seattle (Washington), USA (2014).

5. Poole, E., Chetty, M., Grinter, R., Edwards, W..: More Then Meets the Eye: Transforming the User Experience of Home Network Management. DIS 2008. pp. 455-464 (2008).

6. Björkskog, C.: Human Computer Interaction in Smart Homes. (2007).

7. Takayama, L., Pantofaru, C., Robson, D., Soto, B., Barry, M.: Making Technology Homey: Finding Sources of Satisfaction and Meaning in Home Automation. UbiCom'12., Pittsburgh PA USA (2012).

8. Maloney, J., Resnick, M., Rusk, N., Silverman, B., Eastmond, E.: The Scratch Programming language and Environment. ACM Trans. Comput. Edication. 10, (2010).

9. García-Herranz, M., Haya, P., Alamán, X.: towards a Ubiquitous End-user Programming System for Smart Spaces. J. Univers. Comput. Sci. 16, 1633-1649 (2010).

10. Ur, B., McManus, E., Pak Yong Ho, M., Littman, M.L.: Pratical Trigger-Action Programming in the Smart Home. CHI 2014. pp. 803-812. , Toronto, Ontario, Canada (2014).

11. Demeure, A., Caffiau, S., Coutaz, J.: Activity based End-User-Development for Smart Homes: Relevance and Challenges. Workshop HyperCities (Intelligent Environments). pp. 141-152., Shanghai (2014).

12. Davidoff, S., Lee, M.K., Yiu, C., Zimmerman, J., Dey, A.K.: Principles of Smart Home Control. Ubicomp 2006. pp. 19-34 (2006). 